\title{
Chromosomal instability and aneuploidy: a conundrum in cancer evolution
}

\author{
Jasmin Ali
}

\begin{abstract}
Chromosomal instability (CIN), defined as an increased rate of gain or loss of whole chromosomes, leads to aneuploid cells, which are cells that display an abnormal number of chromosomes. Both CIN and aneuploidy are hallmarks of cancer, yet the underlying mechanisms of CIN and aneuploidy and their impact on tumourigenesis have remained poorly defined. Although multiple mechanisms have been proposed to explain the role of CIN and aneuploidy in tumourigenesis, this review focuses on three principal pathways leading to CIN: spindle assembly checkpoint defects, merotelic attachments, and cohesion defects. Here, we provide a brief overview of the current understanding of the roles of these mechanisms in CIN and aneuploidy. We also present emerging evidence that contradicts the importance of certain mechanisms in cancer evolution. A clearer understanding of these fundamental pathways could prove to be helpful in developing effective cancer therapies.
\end{abstract}

Keywords: Chromosomal instability (CIN); Aneuploidy; Cancer; Spindle assembly checkpoint (SAC); Merotelic attachment; Cohesion defect; Tumour suppressor

\section{Résumé}

L'instabilité chromosomique (INC) est définie comme étant un taux accru de gain ou de perte de chromosomes entiers, qui mène à la formation de cellules aneuploïdes, des cellules avec un nombre anormal de chromosomes. Tous deux I'INC et l'aneuploïdie sont des caractéristiques du cancer, mais leurs mécanismes sous-jacents et leur impact sur la tumourogenèse demeurent ambigus. Bien que plusieurs mécanismes aient été proposés pour expliquer le rôle de I'INC et de l'aneuploïdie dans la tumourogenèse, cet article se concentre sur trois voies principales conduisant à I'INC : les défauts de points de contrôle de l'assemblage du fuseau mitotique, les attachements méroteliques et les défauts de cohésion. Ici, nous fournissons un bref aperçu de la compréhension actuelle des rôles de ces mécanismes impliqués dans I'INC et l'aneuploïdie. Nous présentons également des preuves émergentes qui contredisent l'importance de certains mécanismes dans l'évolution du cancer. Une meilleure compréhension de ces voies fondamentales pourrait s'avérer utile dans le développement de thérapies anticancéreuses efficaces.

Mots Clés: Instabilité chromosomique (INC); Aneuplö̈die; Cancer; Point de contrôle de l'assemblage du fuseau mitotique; Attachement mérotelique; Défaut de cohésion; Suppresseur de tumeur

\section{Introduction}

In any given species, a non-cancerous cell has a defined number of chromosomes with a characteristic genome. It is essential for cells to maintain this genomic integrity during cell division to produce properly functioning daughter cells. A failure in this process can lead to chromosomal instability (CIN), a type of genomic instability, which involves a high rate of gain or loss of whole chromosomes (1). Consequently, daughter cells present an abnormal number of chromosomes, a state referred to

Correspondence: jali086@uottawa.ca

Department of Biology, University of Ottawa, 30 Marie Curie, K1N 6N5,

Ottawa, Canada as aneuploidy. Both CIN and aneuploidy are hallmarks of cancer $(2,3)$.

Over a century ago, German zoologist Theodor Boveri examined sea urchin embryos that underwent abnormal mitotic divisions. Based on his own observations as well as those previously made by David Hansemann, Boveri hypothesized that aneuploidy could cause cancer (4). Boveri's hypothesis became the foundation on which many of the current mechanisms have been theorized. Over the decades, the consistent presence of aneuploid cells in cancers has amplified the belief that CIN and aneuploidy induce tumourigenesis (5). Nevertheless, the mechanisms underlying CIN and aneuploidy and how 
they enhance tumour formation are not yet fully understood. Currently, research has been investigating some of the control mechanisms associated with CIN.

To ensure proper chromosome segregation during cell division, eukaryotes have multiple programmed checks and controls. The spindle assembly checkpoint (SAC) is one of the main control mechanisms. In mammals, the SAC consists of multiple conserved proteins that coordinate with one another to prevent aneuploidy (6). Accordingly, it has been observed that a problem with the SAC can lead to CIN. Moreover, when spindle fibers bind to kinetochores in a specific, but improper configuration, they can escape SAC detection. These merotelic attachments can ultimately lead to the formation of aneuploid cells $(7,8)$. Chromosomal mis-segregation can also arise when there is a flaw in the cohesin complex, which is an important regulator that ensures the correct timing of chromosome division (9). Overall, these regulatory processes involve the cooperation of multiple factors to ensure accurate chromosome segregation (10). A defect in these control mechanisms can result in CIN and aneuploidy, thereby favouring cancer progression (11).

In this review, we provide an overview of the roles of some of the recurring elements in these mechanisms which include the spindle assembly checkpoint, kinetochore-spindle fibre attachments, and chromosome cohesion. We briefly review how disruptions in these key constituents can cause CIN and aneuploidy. Furthermore, we present recent findings that oppose the importance of these mechanisms in tumourigenesis. We offer a possible explanation for these conflicting perspectives by exploring aneuploidy as a tumour suppressor. Future research on these paradigms may help to better explain the role of CIN and aneuploidy in tumourigenesis. A profound understanding of these mechanisms could have an important impact on the development of cancer treatments.

\section{On the highway to CIN}

Throughout eukaryotic evolution, the pathways associated with accurate chromosome transmission have remained conserved and much of the knowledge about CIN and aneuploidy has been acquired by studying model organisms, such as mice and yeast (12). Although multiple mechanisms have been proposed to explain CIN, here we focus on some of the main contributors: a) defects in the $\mathrm{SAC}$ b) merotelic attachments and c) cohesion defects.

\section{Defective spindle assembly checkpoint}

The SAC is a major regulatory pathway of the cell cycle that monitors chromosome division. The SAC ensures all chromosomes are correctly attached to the spindle before entering anaphase. Spindle microtubules attach to the kinetochores, which are protein complexes that assemble at each centromere of the chromosome (13). Normally, unattached kinetochores will signal the SAC to cease mitosis, preventing the loss or gain of a chromosome in a daughter cell (14).

On a molecular level, the SAC is comprised of multiple proteins that arrest mitosis. Some of the conserved SAC proteins include the 'mitotic-arrest deficient' (Mad) proteins Mad1, Mad2, and Mad3 and the 'budding uninhibited' by benzimidazole (Bub) proteins Bub1, BubR1, Bub3 (14). The mitotic checkpoint complex (MCC) is formed when Mad3/BubR1, Mad2, and Bub3 associate with Cdc20, an activator of the anaphase-promoting complex/cyclosome (APC/C). The subsequent conformational change of the APC inhibits its E3 ubiquitin ligase activity, thereby delaying the onset of anaphase (15). This delay allows for chromosomes to achieve the proper bipolar spindle attachment, ensuring the fidelity of chromosome segregation during mitosis (16).

Unsurprisingly, defective SAC components have been associated with CIN and cancer. Studies with testicular germ cell tumours showed reduced Mad2 protein expression in 6 of 8 cell lines (17). The identification of mutations in the Mad2 gene in a breast cancer line and in numerous gastric cancer tumour cell lines corroborated this finding (18). In another case, the mutational inactivation of a human homologue of the $B u b 1$ gene was found in cancer cells presenting CIN (19). All these findings imply mitotic checkpoint defects are necessary for the progression of CIN and tumourigenesis. However, further experiments deem the relationship between SAC defects and tumourigenesis to be more intricate.

The complexity in the correlation between SAC defects and cancer development has been investigated by experiments with mice. Mouse models demonstrate that while homozygous deletion of SAC genes is lethal, mice heterozygous for the genes are viable and have an increased chromosomal mis-segregation rate (18). Although this finding suggests that defective SAC genes are linked with CIN, it has been observed that only some of the heterozygous mice spontaneously form tumours later in life, while other mice that are heterozygous for $B u b 1, B u b R 1$, or $B u b 3$ remain tumour-free (20). Interestingly, when the two heterozygous groups are compared, both groups have similar levels of aneuploidy in spleen cells and mouse embryonic fibroblasts (18). Such observations put into question the association of CIN and tumourigenesis with $\mathrm{SAC}$ defects. Indeed, further research has demonstrated that multiple cancer cells have functional mitotic checkpoints, despite displaying CIN and aneuploidy (21). This indicates that a defective mitotic checkpoint may not be an integral constituent for the formation of tumours.

\section{Merotelic attachments}

In normal cellular divisions, kinetochores bind with microtubules from opposing spindles. However, merotelic 
attachments occur when a single kinetochore attaches to fibers from both poles of the spindle (10). This kind of improper attachment is common and is usually corrected early in mitosis (7). Unlike other forms of attachment, merotelic attachments create kinetochore tension allowing them to bypass the mitotic checkpoint (8). Studies using oocytes demonstrate that merotelic attachments silence Mad2-signalling which contributes to the inactivation of the mitotic checkpoint and to chromosome missegregation in wild-type MII oocytes (22). When this improper attachment continues into anaphase, it can lead to the presence of lagging chromatids, which followed by cytokinesis, can give rise to aneuploid cells (7). To explain the frequent presence of this type of attachment, several mechanisms have been proposed, amongst which, centrosome amplification correlates strongly with CIN (23). Current research also demonstrates the importance of timely centrosome division, an aspect that was historically overlooked.

Because supernumerary centrosomes can form multipolar spindles, which appear in tumours, it was proposed that multipolar divisions were drivers of CIN and tumourigenesis (24). However, the rarity and the detrimental impact of multipolar divisions to cell proliferation led researchers to conclude that multipolar metaphases are transient (25). Instead, the geometry of these transient intermediates promotes merotelic attachments, and thereby increases the frequency of lagging chromosomes in bipolar anaphase, following centrosome clustering (26). Sure enough, a link between centrosome amplification and merotelic attachments has been observed in vivo (10). To determine if supernumerary chromosomes could lead to aneuploidy in mice, researchers isolated and analyzed epidermal cells. While not a single of the 78 control cells were aneuploid, the cells from mice with centrosome amplification showed 23 aneuploid cells out of 99 cells (23). Research does support the increase of aneuploid cells with supernumerary centrosomes. In humans, the presence of abnormal centrosome numbers has been observed in various malignant tumours and has more notably been abundant in high grade metastatic breast adenocarcinoma (27). Consequently, centrosome amplification and merotelic attachments continue to be probable mechanisms leading to CIN.

In addition to centrosome amplification, the timing of centrosome division is becoming an increasingly significant factor in understanding CIN and aneuploidy. Centrosome separation is a regulated mechanism that commences early in the cell cycle to ensure the bipolar spindle accurately divides the duplicated chromosome amongst the two daughter cells (28). Initial work by Silkworth and Cimini indicated that delayed centrosome separation allowed a geometry in which two spindle poles were proximal to one another, thus favouring merotelic attachments (29). Recent research, however, provides a contradictory observation. Studies with mice revealed overexpressed Cyclin B2 hyperactivates aurora-A-mediated Plk1, which causes accelerated centrosome separation and a recurrence of lagging chromosomes (28). Although this study suggests that an accelerated centrosome separation favours an increase in merotelic formation, the mechanism remains to be elucidated. Considering that both, a delay and an acceleration of centrosome division increases frequency of merotelic attachments, it emphasizes the importance of this factor in CIN.

\section{Cohesion defects}

Cohesion plays an integral role in proper genome transmission. Along with serving a purpose in DNA repair and gene expression, cohesion prevents the premature segregation by tethering sister chromatids together from $\mathrm{S}$ phase until the metaphase-to-anaphase transition $(30,31)$.

In a properly segregating cell, cohesion between the sister chromatids remains intact through the G2 and M phases of the cell cycle and then dissolves at anaphase onset following SAC activation (18). Cohesion is achieved by the evolutionarily conserved cohesin complex (32). In humans and mice, the complex is composed of four core subunits. The structural maintenance of chromosomes (Smc) proteins, Smc1 and Smc3, heterodimerize to form a V-shape (9). Another protein, Stag1-3, associates with a kleisin protein, Re8, thereby completing the cohesin complex (33). Defects in cohesion have been observed to cause CIN and aneuploidy.

By sequencing human homologues of genes known to cause CIN in budding yeast, Barber et al. were able to identify mutations in genes responsible for regulating sister chromatid cohesion (12). The Smc1, Smc3, and Stag3 proteins were all found to be mutated in 9 out of 132 colorectal adenocarcinomas (12). Although the direct relevance of these mutations to CIN remains ambiguous, research with Stag2 cultivated a causative link between cohesion defects and CIN. The targeted inactivation of Stag2 in numerous tumours led to defective chromatid cohesion and a rise in aneuploidy, while the targeted correction of mutant alleles resulted in enhanced chromosomal stability (3). Yeast and mice models concurred with these findings $(34,35)$. However, more recent studies demonstrate mixed results and dispute the role of Stag2 in aneuploidy.

Current research with mutated cohesins in naturallyoccurring human tumours indicate little to no correlation to aneuploidy (36). This is supported by the presence of euploid tumours in bladder cancers, despite recurrent inactivation of Stag2 (37). To understand how Stag2 mutations may affect cohesion, Kim et al introduced nine 
tumour-derived mutations, including nonsense and missense mutations into cultured human cells (38). Their findings showed that only the nonsense mutations led to cohesion defects and only one of the nine mutations caused changes in chromosome counts (38). These observations indicate that not all tumour-derived Stag2 mutations have a fundamental role in cohesion defects and aneuploidy. However, the mechanistic basis behind this phenomenon is presently undetermined. In the future, determining the precise functional consequences of different kinds of mutations in cohesion genes could provide insight on their impact in aneuploidy and cancer formation.

\section{Aneuploidy: a double-edged sword}

It has been commonly believed that aneuploidy plays a critical role in cancer progression. Most recently, research has implicated a novel perspective, in which aneuploidy works as a tumour suppressor. Sheltzer et al observed that single-chromosome gains, which consisted of the chromosomes mChr1, mChr13, mChr16, mChr19, hChr3, and hChr5, conferred the trisomic cells a reduced tumourigenicity in comparison to their euploid counterparts (2).

More remarkably, certain aneuploidies have both tumourpromoting and tumour-suppressing characteristics. Such an effect can be observed in Down syndrome (trisomy 21 ) patients. On one hand, children with Down syndrome have an elevated incidence of certain kinds of leukemia such as acute myeloid and lymphoblastic leukemia (39). On the other hand, they exhibit a lower risk of developing most solid tumours (40). Mouse models for trisomy 21 reflect such findings. Studies have shown that the gain of a third copy of the Down syndrome critical region-1 (Dscr 1) gene was sufficient to limit the proliferation of lung tumours by decreasing tumour vascularization and increasing apoptosis of the tumour cells (41).

Because the expression of multiple genes is affected when an extra chromosome is gained, a proposed hypothesis is that the net effect of all gene expression alterations, some of which can be tumour-promoting or tumour-suppressing, dictates whether the aneuploidy is oncogenic or tumour-protective (1). This line of thought could provide insight as to why mechanisms enhance tumour formation in certain contexts, while in other circumstances, they do not appear to play a substantial role. Indeed, it has been discovered that while the surplus of the SAC genes, Mad2 and Bub1, may have tumourigenic effects, the overexpression of BubR1 can counteract defects that affect the mitotic checkpoint and/or kinetochore-microtubule attachments (42). Moreover, mouse embryonic fibroblasts that are trisomic exhibit impaired immortalization and less growth (43). A molecular understanding of such observations could be exploited in the future to advance aneuploid-specific therapies.

\section{Concluding remarks}

Although CIN and aneuploidy have been observed for over a century, research has only begun characterizing the roles of these mechanisms at the molecular level. In doing so, it is apparent that the relationship between CIN, aneuploidy, and tumourigenesis is more complex than what was previously hypothesized. In this review, we summarized literature studying how defects in the SAC, in microtubule-kinetochore attachments, and in the cohesin complex can be sources of CIN and tumourigenesis. We highlighted evidence that contradicted these findings. Lastly, to reconcile these two opposing views, we presented how aneuploidy can be both tumourpromoting and tumour-protective under certain conditions. In future work, it may be useful to revisit how $\mathrm{SAC}$ genes influence the expression of one another. SAC genes have been associated with multiple cellular processes. Could certain contexts cause the expression of certain SAC genes to silence the expression of others? How would this affect CIN and cancer evolution? Are centrosome amplification and centrosome division interconnected? If so, how do supernumerary centrosomes affect centrosome dynamics or vice-versa? At what point during tumourigenesis and in which environments do Stag2 mutations occur? Further investigations into these areas could provide useful information to devise alternative strategies to combat cancer.

\section{Competing interests}

The author declares that she has no competing interests.

Acknowledgements

This was work was supported in part by NSERC grant number 000000-1999

References

1. M. Giam, G. Rancati, Cell Div 10, 3 (2015).

2. J. M. Sheltzer, et al., Cancer Cell 31, 240 (2017)

3. D. A. Solomon, et al., Science 333, 1039 (2011).

4. A. J. Holland, D. W. Cleveland, Nat. Rev. Mol. Cell Biol. 10, 478 (2009).

5. H. Rajagopalan, C. Lengauer, Nature 432, 338 (2004).

6. F. Marchetti, S. Venkatachalam, Cell Cycle 9, 58 (2010).

7. J. Gregan, S. Polakova, L. Zhang, I. M. Tolić-Nørrelykke, D. Cimini, Trends Cell Biol. 21, 374 (2011).

8. A. A. Guerrero, C. Martinez-A, K. H. van Wely, Cell Div 5, 13 (2010).

9. T. Gligoris, J. Lowe, Trends Cell Biol. 26, 680 (2016).

10. D. J. Gordon, B. Resio, D. Pellman, Nat. Rev. Genet. 13, 189 (2012)

11. K. Tanaka, T. Hirota, Biochim. Biophys. Acta 1866, 64 (2016).

12. T. D. Barber, et al., Proc. Natl. Acad. Sci. U.S.A. 105, 3443 (2008).

13. P. Lara-Gonzalez, F. G. Westhorpe, S. S. Taylor, Curr. Biol. 22, R966 (2012).

14. T. Lischetti, J. Nilsson, Mol Cell Oncol 2, e970484 (2015).

15. F. Herzog, et al., Science 323, 1477 (2009).

16. G. J. Gorbsky, FEBS J. 282, 2471 (2015).

17. M. K. Fung, et al., Biochim. Biophys. Acta 1773, 821 (2007)

18. S. L. Thompson, S. F. Bakhoum, D. A. Compton, Curr. Biol. 20, R285 (2010).

19. D. P. Cahill, et al., Nature 392, 300 (1998). 
20. M. E. Burkard, B. A. Weaver, Cancer Discov 7, 134 (2017).

21. B. D. Vitre, D. W. Cleveland, Curr. Opin. Cell Biol. 24, 809 (2012).

22. A. Kouznetsova, A. Hernandez-Hernandez, C. Hoog, Nat Commun 5, 4409 (2014).

23. M. S. Levine, et al., Dev. Cell 40, 313 (2017).

24. A. Krämer, B. Maier, J. Bartek, Mol Oncol 5, 324 (2011)

25. S. A. Godinho, D. Pellman, Philos. Trans. R. Soc. Lond., B, Biol. Sci. 369 (2014).

26. N. J. Ganem, S. A. Godinho, D. Pellman, Nature 460, 278 (2009).

27. J. Y. Chan, Int. J. Biol. Sci. 7, 1122 (2011).

28. H. J. Nam, J. M. van Deursen, Nat. Cell Biol. 16, 538 (2014).

29. W. T. Silkworth, I. K. Nardi, R. Paul, A. Mogilner, D. Cimini, Mol. Biol. Cell 23, 401 (2012)

30. K. Nasmyth, Nat. Cell Biol. 13, 1170 (2011).

31. D. Dorsett, Chromosoma 116, 1 (2007).

32. J. Gerton, PLoS Biol. 3, e94 (2005).

33. J. M. Cheng, Y. X. Liu, Int J Mol Sci 18, 1578 (2017)

34. S. Covo, C. M. Puccia, J. L. Argueso, D. A. Gordenin, M. A. Resnick, Genetics 196, 373 (2014).

35. S. Remeseiro, et al., EMBO J. 31, 2076 (2012).

36. V. K. Hill, J. S. Kim, T. Waldman, Biochim. Biophys. Acta 1866, 1 (2016).

37. C. Balbás-Martinez, et al., Nat. Genet. 45, 1464 (2013).

38. J. S. Kim, et al., PLoS Genet. 12, e1005865 (2016).

39. C. M. Zwaan, D. Reinhardt, J. Hitzler, P. Vyas, Hematol. Oncol. Clin. North Am. 24, 19 (2010)

40. D. Nižetić, J. Groet, Nat. Rev. Cancer 12, 721 (2012).

41. S. Ryeom, et al., Cancer Cell 13, 420 (2008).

42. D. J. Baker, et al., Nat. Cell Biol. 15, 96 (2013).

43. B. R. Williams, et al., Science 322, 703 (2008) 